\title{
A simple method to construct exact density-potential pairs from a homeoidal expansion ${ }^{\star}$
}

\author{
L. Ciotti ${ }^{1}$ and G. Bertin ${ }^{2}$ \\ ${ }^{1}$ Dipartimento di Astronomia, Università di Bologna, via Ranzani 1, 40127 Bologna, Italy \\ e-mail: luca.ciotti@unibo.it \\ 2 Dipartimento di Fisica, Università di Milano, via Celoria 16, 20133 Milano, Italy \\ e-mail: giuseppe.bertin@unimi.it
}

Received 6 October 2004 / Accepted 30 March 2005

\begin{abstract}
We start from a study of the density-potential relation for classical homeoids in terms of an asymptotic expansion for small deviations from spherical symmetry. We then show that such expansion is a useful device that allows us to construct a variety of exact density-potential pairs with spheroidal, toroidal, or triaxial shapes for which the deviation from spherical symmetry is finite. As concrete analytical applications, we describe: (1) The construction of a family of toroidal axisymmetric density-potential pairs one of which is associated with a perfectly flat rotation curve (for a member of this family, the supporting two-integral phase-space distribution function is obtained in closed form); (2) The determination of the aperture velocity dispersion in a wide class of two-integral axisymmetric models not stratified on homeoids with central black hole, which may be useful for the discussion of the dynamical contributions to the characteristics of the Fundamental Plane of early-type galaxies; and (3) For such class of models, the construction of the $v / \sigma$-ellipticity relation, often considered to assess the role of rotation in the structure of elliptical galaxies.
\end{abstract}

Key words. galaxies: kinematics and dynamics - galaxies: structure

\section{Introduction}

For the discussion of many astrophysical problems where gravity is important, a major difficulty is set by the potential theory. In general, to calculate the gravitational potential associated with a given density distribution one has to evaluate a threedimensional integral. Except for special circumstances, where a solution can be found in terms of elementary functions, one has to resort to numerical techniques and sophisticated tools, such as expansions in orthogonal functions or integral transforms.

Under spherical symmetry, the density-potential relation can be reduced to a one-dimensional integral, while for axisymmetric systems one is left in general with a (usually non-trivial) two-dimensional integral. As a result, the majority of explicit density-potential pairs refers to spherical symmetry and only a handful of axially symmetric pairs are known (e.g., see Binney $\&$ Tremaine 1987, hereafter BT87). In few special cases (in particular, when a density-potential pair is available in a suitable parametric form) there exist systematic procedures to generate new non-trivial density-potential pairs (e.g., see the case of Miyamoto \& Nagai 1975 and the related Satoh 1980 disks; see also Evans \& de Zeeuw 1992).

For non-axisymmetric systems the situation is worse. One class of triaxial density distributions for which the potential can

* All Appendices are only available in electronic form at http://www . edpsciences.org be expressed in a tractable integral form is that of the stratified homeoids, such as the Ferrers (1887) distributions (e.g., see Pfenniger 1984; Lanzoni \& Ciotti 2003, hereafter LC03) and special cases of the family considered by de Zeeuw \& Pfenniger (1988). Additional explicit density-potential pairs are given by the Evans (1994) models and by those constructed with the Kutuzov-Osipkov (1980) method (see also Kutuzov 1998).

In this paper we draw attention to an elementary yet curious property of the asymptotic expansion for small flattening of the homeoidal potential quadrature formula. Such expansion can be traced back to the treatise on geodesy by Sir H. Jeffreys (1970, and references therein; see also Hunter 1977). Recently, it has been applied to the modeling of gaseous halos in clusters (Lee \& Suto 2003, 2004) and to the study of the dynamics of elliptical galaxies (Muccione \& Ciotti 2003, 2004, hereafter MC03, MC04). The apparently unnoticed property of the expansion is that it offers a device to construct, in a systematic way, density-potential pairs with finite deviations from spherical symmetry. In turn, these can be used to carry out a number of calculations explicitly, thus allowing for a variety of interesting applications to stellar dynamics.

The paper is organized as follows. In Sect. 2 we present the basic asymptotic expansions and we outline the method. In Sect. 3 we study the Jeans equations for axisymmetric fluid models associated with density distributions obtained from 
our method. In Sect. 4 we focus on three significant applications. In Sect. 5 we make some concluding remarks. In Appendix A we address the issue of the relevant parameter space. In Appendix B we clarify the relation between the homeoidal expansion and the expansion in spherical harmonics. In Appendix $\mathrm{C}$ we summarize the intrinsic kinematical profiles of a family of axisymmetric galaxy models with central black hole.

\section{The method}

We start by assuming a density distribution stratified on homeoidal surfaces, $\rho(\boldsymbol{x})=\rho_{0} \tilde{\rho}(m)$, where $\boldsymbol{x}=(x, y, z), \rho_{0}$ is a density scale, and

$m^{2}=\frac{x^{2}}{a^{2}}+\frac{y^{2}}{b^{2}}+\frac{z^{2}}{c^{2}}=\frac{x^{2}}{a^{2}}+\frac{y^{2}}{a^{2}(1-\epsilon)^{2}}+\frac{z^{2}}{a^{2}(1-\eta)^{2}}$,

with $a \geq b \geq c>0, b / a \equiv 1-\epsilon$, and $c / a \equiv 1-\eta$. The prolate case corresponds to $\epsilon=\eta<1$ and the oblate case to $\epsilon=0$ and $0<\eta<1$. In models of astrophysical interest, the density $\rho$ may extend to infinity (with finite or infinite mass) or be truncated. For simplicity, in this paper we discuss only non-truncated density distributions.

It is well known (e.g., see Kellogg 1953; Chandrasekhar 1969) that the (inner) potential associated with a homeoidal density distribution can be written as

$\phi(x)=-\pi a b c \rho_{0} G \int_{0}^{\infty} \frac{\widetilde{\Delta \Psi}(\boldsymbol{x} ; \epsilon, \eta)}{\Delta(\tau)} \mathrm{d} \tau$,

where $\Delta(\tau)=\sqrt{\left(a^{2}+\tau\right)\left(b^{2}+\tau\right)\left(c^{2}+\tau\right)}, \widetilde{\Delta \Psi}=$ $2 \int_{m(\boldsymbol{x} ; \tau)}^{\infty} \tilde{\rho}(m) m \mathrm{~d} m$, and $m^{2}(\boldsymbol{x} ; \tau)=x^{2} /\left(a^{2}+\tau\right)+y^{2} /\left(b^{2}+\tau\right)+$ $z^{2} /\left(c^{2}+\tau\right)$. If we rescale density and potential to the quantities $\rho_{0}$ and $4 \pi G \rho_{0} a^{2}$, respectively, the Poisson equation for the dimensionless density-potential pair $(\tilde{\rho}, \tilde{\phi})$ becomes $\tilde{\nabla}^{2} \tilde{\phi}=\tilde{\rho}$ where $\tilde{\nabla}^{2}=\partial^{2} / \partial \tilde{x}^{2}+\partial^{2} / \partial \tilde{y}^{2}+\partial^{2} / \partial \tilde{z}^{2}, \tilde{x} \equiv x / a, \tilde{y} \equiv y / a$, $\tilde{z} \equiv z / a$ and

$\tilde{\phi}=-(1-\epsilon)(1-\eta) \frac{a}{4} \int_{0}^{\infty} \frac{\widetilde{\Delta \Psi}(\boldsymbol{x} ; \epsilon, \eta)}{\Delta(\tau)} \mathrm{d} \tau$.

This will be the starting point of our analysis. We now expand the density $\tilde{\rho}(m)$ and the potential $\tilde{\phi}$ given in Eq. (3) up to the first significant order in the flattening parameters and find:

$\tilde{\rho}(m)=\tilde{\rho}(\tilde{r})+\frac{\epsilon \tilde{y}^{2}+\eta \tilde{z}^{2}}{\tilde{r}} \tilde{\rho}^{\prime}(\tilde{r})+O\left(\epsilon^{2}+\eta^{2}\right)$,

where $\tilde{\rho}^{\prime} \equiv \mathrm{d} \tilde{\rho}(m) / \mathrm{d} m$ evaluated at $\epsilon=\eta=0$, and

$$
\begin{aligned}
\tilde{\phi}= & \tilde{\phi}_{0}(\tilde{r})+(\epsilon+\eta)\left[\tilde{\phi}_{1}(\tilde{r})-\tilde{\phi}_{0}(\tilde{r})\right]+\left(\epsilon \tilde{y}^{2}+\eta \tilde{z}^{2}\right) \tilde{\phi}_{2}(\tilde{r}) \\
& +O\left(\epsilon^{2}+\eta^{2}\right),
\end{aligned}
$$

where

$$
\tilde{\phi}_{i}(\tilde{r})=\left\{\begin{array}{l}
-\frac{1}{\tilde{r}} \int_{0}^{\tilde{r}} \tilde{\rho}(m) m^{2} \mathrm{~d} m-\int_{\tilde{r}}^{\infty} \tilde{\rho}(m) m \mathrm{~d} m, \quad(i=0) \\
-\frac{1}{3 \tilde{r}^{3}} \int_{0}^{\tilde{r}} \tilde{\rho}(m) m^{4} \mathrm{~d} m-\frac{1}{3} \int_{\tilde{r}}^{\infty} \tilde{\rho}(m) m \mathrm{~d} m,(i=1) ; \\
\frac{1}{\tilde{r}^{5}} \int_{0}^{\tilde{r}} \tilde{\rho}(m) m^{4} \mathrm{~d} m, \quad(i=2) .
\end{array}\right.
$$

In the above expressions, the dimensionless spherical radius is defined as $\tilde{r} \equiv r / a=\sqrt{\tilde{x}^{2}+\tilde{y}^{2}+\tilde{z}^{2}}$. Note that $\tilde{\phi}_{0}(\tilde{r})$ is the potential associated with $\tilde{\rho}(\tilde{r})$, as can be seen from its explicit expression. The infinite upper limit of integration appearing in two integrals could be changed to a finite constant if desired; this property is useful to deal with density distributions for which the two integrals would diverge. Similar formulae can be obtained for the expansion at fixed total mass of density distributions of finite total mass (e.g., see MC04, Eq. (C.1)).

\subsection{Generating new families of non-spherical density-potential pairs}

By ordering arguments and by the linearity of the Poisson equation, it follows that the truncation of Eqs. (4) and (5) to first order in the flattening parameters produces exact densitypotential pairs independently of the value of $\epsilon$ and $\eta$. In order to be physically acceptable, the density distribution truncated to first order in $\epsilon$ and $\eta$ must be positive definite (see Appendix A). Density-potential pairs belonging to the families thus constructed can be non-spherical at the "non-linear" level, because the values of the flattening parameters can differ significantly from zero. On the other hand, the truncated potential is also the approximate potential for the density $\rho=\rho_{0} \tilde{\rho}(m)$ when $\epsilon \rightarrow 0$ and $\eta \rightarrow 0$.

Now we proceed one step further and use Eqs. (4) and (5) to construct new density-potential pairs, independent of the ellipticities $\epsilon$ and $\eta$ defining the homeoidal expansion. In fact, by the same ordering argument used above, it is immediate to see that $\tilde{\phi}_{1}(\tilde{r})-\tilde{\phi}_{0}(\tilde{r})+\tilde{z}^{2} \tilde{\phi}_{2}(\tilde{r})$ is the potential associated with the "density" $\tilde{z}^{2} \tilde{\rho}^{\prime}(\tilde{r}) / \tilde{r}$. For radially declining density distributions $\tilde{\rho}(\tilde{r})$, we can thus consider the (dimensionless) pair

$\varrho=\tilde{z}^{2} \frac{\left|\tilde{\rho}^{\prime}(\tilde{r})\right|}{\tilde{r}} ; \quad \Phi=\tilde{\phi}_{0}-\tilde{\phi}_{1}-\tilde{z}^{2} \tilde{\phi}_{2}$.

Two similar density-potential pairs can be then obtained trivially by renaming variables, i.e., by replacing $\tilde{z}$ with $\tilde{y}$ or $\tilde{x}$ in Eq. (7). We now combine the three density-potential pairs linearly to get the following generally "triaxial" solution

$\varrho=\left(\alpha \tilde{x}^{2}+\beta \tilde{y}^{2}+\gamma \tilde{z}^{2}\right) \frac{\left|\tilde{\rho}^{\prime}(\tilde{r})\right|}{\tilde{r}}$,

$\Phi=(\alpha+\beta+\gamma)\left[\tilde{\phi}_{0}(\tilde{r})-\tilde{\phi}_{1}(\tilde{r})\right]-\left(\alpha \tilde{x}^{2}+\beta \tilde{y}^{2}+\gamma \tilde{z}^{2}\right) \tilde{\phi}_{2}(\tilde{r})$.

If we now set $\alpha=\beta=1$ and $\gamma=0$, we find a new axisymmetric family characterized by toroidal geometry

$\varrho=\tilde{R}^{2} \frac{\left|\tilde{\rho}^{\prime}(\tilde{r})\right|}{\tilde{r}} ; \quad \Phi=2\left(\tilde{\phi}_{0}-\tilde{\phi}_{1}\right)-\tilde{R}^{2} \tilde{\phi}_{2}$

Here $\tilde{R} \equiv \sqrt{\tilde{x}^{2}+\tilde{y}^{2}}$ is the dimensionless cylindrical radius.

That Eqs. (7)-(10) indeed identify explicit densitypotential pairs can be checked a posteriori by deriving the potential $\Phi$ from the density $\varrho$ by means of a standard expansion in spherical harmonics (Appendix B). Note however that, if we started with a seemingly simpler idea, i.e., to transform the density $\tilde{\rho}(m)$ in spherical coordinates and then to expand it in spherical harmonics, we would have discarded all the expansion terms above the monopole as candidates for acceptable 
density distributions because the related mass vanishes. In contrast, the present method works because, at least to first order, it is possible to isolate a density term with constant (in our case, negative) sign over all the space.

In the approach followed so far, we have considered the density $\rho=\rho_{0} \tilde{\rho}(m)$ as given: in this way, several densitypotential pairs can be easily obtained, for example by starting from well known seeds for $\tilde{\rho}(\tilde{r})$, such as the density of the $\gamma$-models (Dehnen 1993; Tremaine et al. 1994, see also Appendix A). However, the generated density profiles given in Eqs. (7)-(10) remain largely out of control.

On the other hand, it is possible to specify the spherically symmetric factor $\widehat{\rho} \equiv\left|\tilde{\rho}^{\prime}(\tilde{r})\right| / \tilde{r}$ in Eqs. (7), (8), and (10): the potentials $\tilde{\phi}_{0}, \tilde{\phi}_{1}$, and $\tilde{\phi}_{2}$ required to complete the non-spherical density-potential pair are recovered by inserting in Eq. (6) the supporting density

$\tilde{\rho}(\tilde{r}) \equiv \int_{\tilde{r}}^{\infty} \widehat{\rho}(t) t \mathrm{~d} t$

The function $\widehat{\rho}$ should go to zero sufficiently fast when $r \rightarrow \infty$, in order for the integrals appearing in Eq. (11) to converge. We will use this approach to build the special model presented in Sect. 4.1.

Another way to specify the supporting density $\tilde{\rho}$ (and so $\widehat{\rho}$ ) is by imposing a given rotation curve in the equatorial plane, that is a circular velocity $\tilde{v}_{\mathrm{c}}(\tilde{R})$ defined from the relation $\tilde{v}_{\mathrm{c}}^{2}(\tilde{R}) \equiv$ $v_{\mathrm{c}}^{2}(R) /\left(4 \pi G \rho_{0} a^{2}\right)=\tilde{R} \partial \Phi / \partial \tilde{R}$ at $z=0$. In fact, by considering the dimensionless potential $\Phi$ in Eq. (10) and by assigning the function $\tilde{v}_{\mathrm{c}}^{2}$, one gets and can solve the following (inhomogeneous Euler) differential equation for $\tilde{\rho}$ :

$\tilde{R}^{4} \frac{\mathrm{d}^{2} \tilde{\rho}}{\mathrm{d} \tilde{R}^{2}}+6 \tilde{R}^{3} \frac{\mathrm{d} \tilde{\rho}}{\mathrm{d} \tilde{R}}+2 \tilde{R}^{2} \tilde{\rho}+\frac{\mathrm{d}}{\mathrm{d} \tilde{R}}\left[\frac{1}{\tilde{R}} \frac{\mathrm{d}}{\mathrm{d} \tilde{R}}\left(\tilde{R}^{3} \tilde{v}_{\mathrm{c}}^{2}\right)\right]=0$.

\section{The Jeans equations of stellar dynamics for axisymmetric density-potential pairs (embedded, if desired, in a spherical external field)}

In this section, in view of applications to galactic dynamics, we study the Jeans equations for axisymmetric density-potential pairs either in the form of Eqs. (7) and (10), or in the axisymmetric cases of Eqs. (4) and (5). In our study we can also include the case in which the density distributions mentioned above are added to a separate spherically symmetric density distribution.

In all the axisymmetric cases described above, the pair $(\varrho, \Phi)$ belongs to the general family of density-potential pairs ${ }^{1}$

$\rho_{[R]}=A(r)+R^{2} B(r), \quad \phi_{[R]}=C(r)+R^{2} D(r)$,

where the subscript $[R]$ means that the pair is made explicit with respect to $R$ by using (if required) the identity $z^{2}=r^{2}-R^{2}$. The possibility to switch to an alternative representation (i.e., from $\rho_{[R]}$ and $\phi_{[R]}$ to $\rho_{[z]}$ and $\left.\phi_{[z]}\right)$ is useful for the solution

\footnotetext{
${ }^{1}$ In the self-gravitating case $A(r) \propto C^{\prime \prime}(r)+2 C^{\prime}(r) / r+4 D(r)$ and $B(r) \propto D^{\prime \prime}(r)+6 D^{\prime}(r) / r$.
}

of the Jeans equations (when no subscript appears, it means that either form can be considered). Note that for $D(r) \propto 1 / r^{4}$ the potential belongs to the class of Stäckel potential (Stäckel 1890).

Let us assume that the density-potential pair defines the equilibrium configuration of a stellar dynamical system for which the underlying distribution function (DF) depends on the two classical integrals $E$ and $J_{z}$ (star energy and $z$-component of the angular momentum, per unit mass), so that the velocity dispersion tensor $\sigma_{i j}$ is diagonal and $\sigma^{2} \equiv \sigma_{R R}=\sigma_{z z}$; we will denote by $\sigma_{\varphi}^{2}$ the dispersion $\sigma_{\varphi \varphi}$. Along the azimuthal direction, part of the kinetic energy can be stored in systematic motions ${ }^{2}$, so that $\overline{v_{\varphi}^{2}}=u_{\varphi}^{2}+\sigma_{\varphi}^{2}$.

Given the form of the density-potential pair in Eq. (13), the Jeans equations (e.g., see LC03, Eqs. (7) and (8)) can be easily solved as

$\rho \sigma^{2}=\int_{z}^{\infty} \rho \frac{\partial \phi}{\partial z^{\prime}} \mathrm{d} z^{\prime}=\int_{r}^{\infty} \rho_{[R]} \frac{\partial \phi_{[R]}}{\partial r^{\prime}} \mathrm{d} r^{\prime}$,

and

$$
\begin{aligned}
\rho\left(\overline{v_{\varphi}^{2}}-\sigma^{2}\right) & =R\left(\frac{\partial \rho \sigma^{2}}{\partial R}+\rho \frac{\partial \phi}{\partial R}\right) \\
& =\frac{R^{2}}{r}\left[\frac{\partial\left(\rho \sigma^{2}\right)_{[z]}}{\partial r}+\rho \frac{\partial \phi_{[z]}}{\partial r}\right] .
\end{aligned}
$$

Clearly an ambiguity remains as to the amount of support related to systematic motions with respect to that of random motions in the toroidal direction. A commonly used decomposition assumes $u_{\varphi}^{2} \equiv k^{2}\left(\overline{v_{\varphi}^{2}}-\sigma^{2}\right)$, and so $\sigma_{\varphi}^{2}=\sigma^{2}+\left(1-k^{2}\right)\left(\overline{v_{\varphi}^{2}}-\sigma^{2}\right)$, where $k$ is generally taken to be a given constant (Satoh 1980; but see Ciotti \& Pellegrini 1996). When $k=0$ the system is azimuthally supported only by velocity dispersion, and no net rotation is present; in the opposite limit, $k=1$, the velocity dispersion is isotropic. Of course, the above procedure can be applied only when $\overline{v_{\varphi}^{2}}-\sigma^{2} \geq 0$ everywhere.

The general expression of projection integrals for axisymmetric systems can be found in LC03. For simplicity, in this paper we focus on the case when the system is observed edgeon (i.e. from a line-of-sight where the contribution of rotation is expected to be largest), even though for density distributions as in Eq. (13) explicit projection formulae can be obtained for any inclination of the line-of-sight direction $\boldsymbol{n}$. Thus, we project along the $x$ axis, so that $\boldsymbol{n}=(1,0,0)$ and the projection plane is the $(y, z)$ plane. The projected density is given by $\Sigma=\int_{-\infty}^{\infty} \rho \mathrm{d} x$, while the mass-weighted projected streaming velocity field is given by $\Sigma u_{\mathrm{p}}=\int_{-\infty}^{\infty} \rho u_{\mathrm{n}} \mathrm{d} x$, where $u_{\mathrm{n}} \equiv u_{i} n_{i}=u_{x}=-u_{\varphi} \sin \varphi$ and $\sin \varphi=y / R$; similarly, the projection of the square of the streaming velocity field is $\Sigma V_{\mathrm{p}}^{2} \equiv \int_{-\infty}^{\infty} \rho u_{\mathrm{n}}^{2} \mathrm{~d} x$. Finally, the line-of-sight velocity dispersion field is $\sigma_{\text {los }}^{2}=\sigma_{\mathrm{p}}^{2}+V_{\mathrm{p}}^{2}-u_{\mathrm{p}}^{2}$, where $\Sigma \sigma_{\mathrm{p}}^{2} \equiv \int_{-\infty}^{\infty} \rho \sigma_{\mathrm{n}}^{2} \mathrm{~d} x$, and $\sigma_{\mathrm{n}}^{2} \equiv \sigma_{i j} n_{i} n_{j}=\sigma_{x x}=\sigma^{2}+\left(1-k^{2}\right)\left(\overline{v_{\varphi}^{2}}-\sigma^{2}\right) \sin ^{2} \varphi$ (see Eqs. (B5) in $\mathrm{LC} 03$ ).

\footnotetext{
2 A bar over a quantity means average over phase-space velocities. In particular, $u_{\varphi} \equiv \overline{v_{\varphi}}=\int f v_{\varphi} \mathrm{d}^{3} v / \rho$.
} 


\subsection{The problem of the supporting distribution function}

At the end of this section, we may ask whether it would be possible to reconstruct the phase-space DF for the general family of densities given in Eq. (10). As is well known, the DF

$f=f_{0} J_{z}^{2} h(\mathcal{E}) \Theta(\mathcal{E})$,

where $\mathcal{E}=-v^{2} / 2+\Psi$ is the star binding energy per unit mass, and $\Theta$ is the Heaviside step function, produces a mass density given by

$\varrho=\frac{2^{7 / 2} \pi f_{0}}{3} R^{2} \int_{0}^{\Psi}(\Psi-\mathcal{E})^{3 / 2} h(\mathcal{E}) \mathrm{d} \mathcal{E}$

without streaming velocity (e.g., Hunter 1977; for a more general family of distribution functions containing Eq. (16) as a special case, see also Ciotti et al. 2004). Equation (17) can be used to determine the two-integral DF associated with a density profile of the form given by Eq. (10), in the limiting case when the total potential $-\Psi$ is dominated by an external, spherically symmetric potential $-\Psi_{\text {ext }}(r)$ (so that, in such approximation, we ignore the contribution to the potential given by the field produced by $\varrho$ itself). In particular, three different strategies can be considered. In the first two cases $h(\mathcal{E})$ is assigned and then Eq. (17) is used to determine either the spherical factor $\widehat{\rho}(r)$ for given $\Psi_{\text {ext }}(r)$, or, alternatively, $\Psi_{\text {ext }}(r)$ for given $\widehat{\rho}(r)$. In the third case, the two functions $\Psi_{\text {ext }}(r)$ and $\widehat{\rho}(r)$ are assigned, and $h(\mathcal{E})$ is obtained by standard Abel inversion of the function $\widehat{\rho}\left[\Psi_{\text {ext }}\right]$. Of course, in each of these cases the appropriate physical requirements must be satisfied, namely the mass density distribution associated with $-\Psi_{\text {ext }}$ via the Poisson equation and the function $h$ must be non-negative. Note that if $\mathrm{d} \widehat{\rho} / \mathrm{d} r \leq 0$ then $r$ provides the natural parameter to obtain the mapping $\Phi=\Phi\left(\varrho, \tilde{R}^{2}\right)$ from Eq. (10). This is an important step, because $\varrho=\varrho\left(\Phi, \tilde{R}^{2}\right)$ is the function needed to recover the two-integral DF of axisymmetric systems (cf. the Fricke (1952) series expansion, the Lynden-Bell (1962), the Dejonghe (1986), and the Hunter (1975) integral transforms, and finally the Hunter \& Qian (1993) integral contour method; see also Sect. 4.1.2).

\section{Three applications}

Following the normalization introduced at the beginning of Sect. 2 , in this section and in Appendix C, the density $\varrho$, the relevant radii, and the potential $\Phi$ are meant to be normalized to $\rho_{0}$, to the scale-length $a$, and to $4 \pi G \rho_{0} a^{2}$, respectively. It follows that masses, projected densities, and velocities are normalized to $\rho_{0} a^{3}, \rho_{0} a$, and $2 a \sqrt{\pi G \rho_{0}}$; for simplicity, we omit the tilde above these quantities.

\subsection{Power-law tori}

In this subsection we describe a toroidal stellar system belonging to the family associated with Eq. (10), for which the general methods outlined in Sect. 3 allow us to obtain explicitly the relevant kinematical profiles (and their projections on the plane of the sky), even in the presence of a central black hole. For simplicity, we focus here on the fully self-gravitating case, i.e. on the case when no black hole is present. The simplest model is that of a power-law torus, defined by the (normalized) density distribution

$\varrho=\frac{\tilde{R}^{2}}{\tilde{r}^{\alpha}}, \quad(\alpha>0)$.

The total mass of the model is infinite, independently of the value of $\alpha$, an unpleasant property shared with spherically symmetric (non-truncated) power-law models. The central mass is finite for $\alpha<5$. According to Eq. (11) the supporting density is $\tilde{\rho}=\tilde{r}^{2-\alpha} /(\alpha-2)$ (for $\alpha>2$ ), and so from Eqs. (10) and (6)

$\Phi=\left\{\begin{array}{l}-\frac{\tilde{r}^{2-\alpha}}{(\alpha-2)(7-\alpha)}\left[\frac{4 \tilde{r}^{2}}{(\alpha-4)(5-\alpha)}+\tilde{R}^{2}\right], \quad(\alpha \neq 4), \\ \frac{1}{3}\left(2 \ln \tilde{r}-\frac{1}{2} \frac{\tilde{R}^{2}}{\tilde{r}^{2}}\right), \quad(\alpha=4) .\end{array}\right.$

For $4<\alpha<5$ the gravitational potential vanishes for $r \rightarrow \infty$ and diverges at the origin: thus, only orbits with negative total energy are bound. For $2<\alpha \leq 4$ an upper truncation must be applied to some integrals appearing in Eq. (6); the additive constant resulting in Eq. (19) has been set equal to zero, and the potential vanishes at the origin. Finally, for $\alpha=4$ the potential diverges both at $r=0$ and $r=\infty$ and so orbits are bound independently of their energy.

For $2<\alpha<5$ and $\alpha \neq 0$ the circular velocity in the equatorial plane is

$v_{\mathrm{c}}^{2}=\frac{\left(-\alpha^{2}+9 \alpha-16\right) \tilde{R}^{4-\alpha}}{(\alpha-2)(5-\alpha)(7-\alpha)}$

while $v_{\mathrm{c}}^{2}=2 / 3$ for $\alpha=4$. Curiously, the circular velocity vanishes for $\alpha=\alpha_{n} \equiv 9 / 2-\sqrt{17} / 2 \approx 2.44$. In fact, for $2<\alpha<\alpha_{n}$ Eq. (20) would predict a negative square circular velocity, in the sense that in this range of $\alpha$ the radial force field in the equatorial plane, is directed outward. For $2<\alpha<5$, Eqs. (14) and (15) give

$\varrho \sigma^{2}=\frac{\tilde{R}^{2} \tilde{r}^{2(1-\alpha)}}{7-\alpha}\left[\frac{2 \tilde{r}^{2}}{(\alpha-2)^{2}(5-\alpha)}+\frac{\tilde{R}^{2}}{2(\alpha-1)}\right]$,

and

$\varrho\left(\overline{v_{\varphi}^{2}}-\sigma^{2}\right)=\frac{2 \tilde{R}^{2} \tilde{r}^{2(1-\alpha)}}{(\alpha-2)(7-\alpha)}\left[\frac{2 \tilde{r}^{2}}{(\alpha-2)(5-\alpha)}-\frac{\tilde{R}^{2}}{\alpha-1}\right]$

since the r.h.s. of Eq. (22) is positive, the Satoh decomposition can be applied.

In the isotropic case $(k=1)$, Eq. (22) gives the square of the streaming velocity $u_{\varphi}^{2}$; in this case in the equatorial plane (where $r=R$ ), the ratios $\sigma^{2} / v_{\mathrm{c}}^{2}, \overline{v_{\varphi}^{2}} / v_{\mathrm{c}}^{2}$, and $u_{\varphi}^{2} / v_{\mathrm{c}}^{2}$ are constant. The isotropic case can also be interpreted as the description of a toroidal fluid structure for which the velocity $u_{\varphi}$ is not constant over cylinders (corresponding to the fact that the system is baroclinic; e.g., see Tassoul 1978): the asymmetric drift $v_{\mathrm{c}}^{2}-u_{\varphi}^{2}$ on the equatorial plane presented by all the models scales as $R^{4-\alpha}$, vanishes for $\alpha=3$, and is negative for lower values of $\alpha$. Along the $z$ axis (where $R=0$ ), according to Eq. (21) 
the pressure vanishes, and so does the quantity $\varrho\left(\overline{v_{\varphi}^{2}}-\sigma^{2}\right)$ : while this latter behavior is a common property of two-integral axisymmetric systems (e.g., see Ciotti \& Pellegrini 1996, LC03), the former is a peculiarity of the present toroidal model. Along the major axis in the edge-on projection plane (i.e., along the $y$ axis, at $z=0), u_{\mathrm{p}} \propto k \tilde{y}^{2-\alpha / 2}$, while $\sigma_{\text {los }}^{2}=\sigma_{\mathrm{p}}^{2}+V_{\mathrm{p}}^{2}-u_{\mathrm{p}}^{2} \propto \tilde{y}^{4-\alpha}$, where the proportionality constants are simple functions of $\alpha$ that can be easily computed for $\alpha>7 / 2$; below this value of $\alpha$ the projected velocity dispersion diverges. The properties of two toroidal models associated with Eq. (18) are illustrated in Fig. 1.

\subsubsection{The torus with flat rotation curve and the "neutral" torus}

From the solution of Eq. (12) with $v_{\mathrm{c}}=$ const., one can prove that the $\alpha=4$ power-law torus is the only model of this class characterized by a perfectly flat rotation curve. For a generic value of $k$, along the major axis in the edge-on projection, we have $u_{\mathrm{p}}^{2}=8 k^{2} /\left(9 \pi^{2}\right)$, and $\sigma_{\text {los }}^{2}=1 / 3-8 k^{2} /\left(9 \pi^{2}\right)$.

A more general family of power-law tori with flat rotation curves can be obtained by expansion of the oblate spheroid $\rho \propto 1 / m^{2}$ (the so-called "isothermal" spheroid) to higher orders in the flattening, beyond Eqs. (4) and (5). In fact, such oblate spheroid is characterized by a flat rotation curve on the equatorial plane (for any flattening; e.g., see BT87, Chap. 2). Therefore, following the arguments presented in Sect. 2.1 we find that the tori

$\varrho=\frac{\tilde{R}^{2 n}}{\tilde{r}^{2 n+2}}, \quad(n$ integer $)$,

all belong to the list of density distributions with perfectly flat rotation curve, which includes the singular isothermal sphere and the Mestel (1963) disk (see also Monet et al. 1981; the toroidal solutions presented by Toomre 1982 can also be expressed as a superposition of power-law tori). When projected face-on, much like the isothermal sphere, the family given in Eq. (23) produces the $1 / R$ surface density distribution of the Mestel disk.

A torus with very unusual characteristics is the following. If we discard one of the two homogeneous solutions of Eq. (12) (corresponding to $v_{\mathrm{c}}=0$ ), because of its infinite central mass, we are left with the solution $\tilde{\rho} \propto \tilde{r}^{-5 / 2+\sqrt{17} / 2}$. From Eqs. (20) and (11) we see that the related density is given as in Eq. (18), with $\alpha=\alpha_{n}$. This torus is thus characterized by a constant potential in the equatorial plane, so that the circular velocity vanishes, as already pointed out in the previous section. From a mathematical point of view, adding this neutral torus to a disk, would not alter the rotation curve in the disk. In other words, if we add the neutral torus to a flat-rotation density distribution, the resulting density distribution will be associated with a flat rotation curve of the same amplitude as the original.

We conclude by noting that fully self-consistent, selfgravitating toroidal stellar systems corresponding to the density-potential pair (18)-(19) with $\alpha \leq \alpha_{n}$ do not exist. In fact, for $2<\alpha \leq \alpha_{n}$ the zero-velocity curves in the meridional plane $(R, z)$ are open. Surprisingly, the Jeans equations would lead to apparently "innocent" solutions even for $\alpha \leq \alpha_{n}$. This
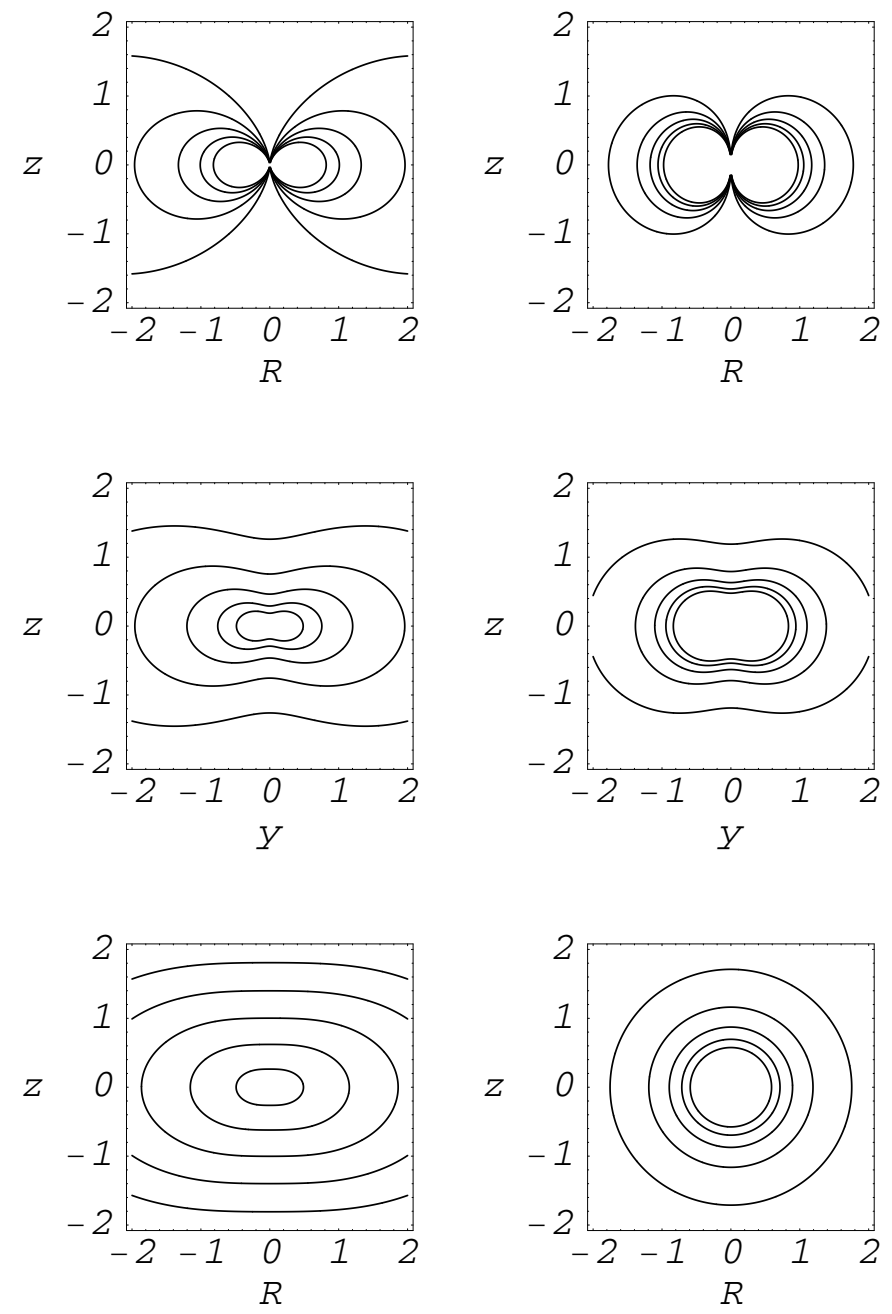

Fig. 1. Isodensity (top), constant projected density (middle), and isopotential (bottom) contours (in arbitrary units) for the toroidal model described by Eq. (18) with $\alpha=3.1$ (left) and $\alpha=4.9$ (right). The lower value of $\alpha$ is near the critical value for which the surface brightness is everywhere infinite, while the upper value is near the critical value for which the central mass diverges. The coordinates $R, y$, and $z$ are normalized to the scale-length $a$. Note how a strongly nonspherical density distribution, such as that represented on the top right, produces a nearly spherical potential (bottom right).

concrete example illustrates the risks that are taken when stellar systems are modeled by means of the Jeans equations without a final check on the physical viability of the solutions found from the formal analysis.

\subsubsection{The two-integral distribution function of power-law tori}

The general considerations presented in Sect. 3.1 can be elaborated in greater detail for the family of power-law tori. In the case of non-self gravitating power-law tori, the distribution function in the presence of a dominant massive central black hole of mass $M_{\mathrm{BH}}$ can be explicitly written as a Fricke distribution function by adopting $h(\mathcal{E})=\mathcal{E}^{\alpha-5 / 2}$ (with $\alpha>3 / 2$ ) and $f_{0}=3 \rho_{0} a^{\alpha-2} /\left[2^{7 / 2} \pi\left(G M_{\mathrm{BH}}\right)^{\alpha} \mathrm{B}(5 / 2, \alpha-3 / 2)\right]$ in Eq. (16), where $\mathrm{B}(x, y)$ is the standard Euler complete Beta function. 
As noted at the end of Sect. 3.1, by combining Eqs. (18) and (19), the function $\Phi=\Phi\left(\varrho, \tilde{R}^{2}\right)$ can be obtained explicitly. In addition, for the entire family of power-law tori (with $\alpha>\alpha_{n}$ ), the envelope and the dynamical window (relevant to such method) can be expressed in terms of elementary functions: for example, the envelope is defined by the relation $J_{z}^{2} \propto \exp \left(3 E_{\mathrm{c}}\right)$ when $\alpha=4$, while $J_{z}^{2} \propto\left|E_{\mathrm{c}}\right|^{\frac{6-\alpha}{4-\alpha}}$ in all the other cases, where $E_{\mathrm{c}}$ is the energy per unit mass of a star orbiting on the circular orbit. Finally, for two members of this family it is possible to construct the function $\varrho=\varrho\left(\Phi, \tilde{R}^{2}\right)$ explicitly, thus providing analytically all the required ingredients for a numerical implementation of the Hunter-Qian method (Qian et al. 1995). In particular,

$\varrho=\left\{\begin{array}{l}\frac{\left(\sqrt{4 \Phi^{2}+2 \tilde{R}^{2}}-2 \Phi\right)^{3}}{\tilde{R}^{4}}, \quad(\alpha=3), \\ \frac{4 W^{2}\left(\tilde{R}^{2} \mathrm{e}^{-3 \Phi} / 2\right)}{\tilde{R}^{2}}, \quad(\alpha=4),\end{array}\right.$

where $W(x)$ is the Lambert function ${ }^{3}$. Remarkably, for $\alpha=3$ the density $\varrho$ can be expanded in series of $\psi^{2} \equiv \tilde{R}^{2} /\left(2 \Phi^{2}\right)$, and the associated series of Fricke's terms can be re-summed in closed form as

$$
\begin{aligned}
f\left(E, J_{z}\right)= & \frac{945}{2^{11 / 2} \pi} \frac{\xi}{E^{5 / 2}}\left[{ }_{2} F_{1}\left(\frac{11}{6}, \frac{13}{6} ; 4,-\frac{27 \xi}{4}\right)\right. \\
& \left.-\frac{143 \xi}{256}{ }_{2} F_{1}\left(\frac{17}{6}, \frac{19}{6} ; 5,-\frac{27 \xi}{4}\right)\right] \\
& +k \operatorname{sign}\left(J_{z}\right) \frac{315}{128 \pi} \frac{\xi}{E^{5 / 2}} \\
& \times{ }_{4} F_{3}\left(\frac{3}{2}, \frac{5}{2}, \frac{11}{6}, \frac{13}{6} ; \frac{5}{4}, \frac{7}{4}, 4,-\frac{27 \xi}{4}\right),
\end{aligned}
$$

which is positive for $0 \leq \xi \equiv J_{z}^{2} /\left(4 E^{3}\right) \leq 32 / 27$ and $0 \leq$ $k \leq 1$ (where the upper limit refers to circular orbits and ${ }_{p} F_{q}$ is the standard hypergeometric function, e.g. see Gradshteyn \& Ryzhik 1980). Note that the odd part has been recovered under the Satoh ansatz, for which $u_{\varphi}=k \sqrt{\Phi}$ from Eqs. (18), (19), and (22). We verified by direct integration over velocity space that the DF returns indeed the $\alpha=3$ torus.

\subsection{Scale-free oblate axisymmetric systems treated in terms of the Jeans equations and the Satoh decomposition}

We now apply the formulae derived in Sect. 2.1 to the family of power-law oblate $(\epsilon=0)$ spheroids. Thus, we start from $\rho=\rho_{0} / m^{\gamma}$, where the range $0<\gamma<3$ is such that the density profile is radially decreasing and the central mass remains finite.

Several properties of these models have been investigated by Qian et al. (1995), who reconstructed their phase-space properties; in addition, Evans (1994) and Evans \& de Zeeuw (1994) studied the observational properties that would result from a decomposition similar (but not identical) to the Satoh (1980) decomposition for a similar class of models

\footnotetext{
3 The Lambert function is defined by the identity $\ln W\left(\mathrm{e}^{z}\right)+W\left(\mathrm{e}^{z}\right)=z$.
}

(which they called "power-law" models, in their scale-free limit).

Here we study the relevant Jeans equations on the basis of the Satoh decomposition, which has found widespread applications to elliptical galaxies (e.g., see Binney et al. 1990; van der Marel et al. 1990; van der Marel 1991). We are then able to provide expressions for the intrinsic and projected kinematical profiles in a form that may be useful to model the observations. These expressions can be easily generalized to the case in which a central black hole or a power-law dark matter halo with different flattening are present.

From Eqs. (4) and (5) we have

$$
\begin{aligned}
& \varrho=\frac{1-\gamma \eta}{\tilde{r}^{\gamma}}+\frac{\gamma \eta \tilde{R}^{2}}{\tilde{r}^{\gamma+2}}, \\
& \Phi=\left\{\begin{array}{l}
-\frac{5-\gamma-(4-\gamma)(\gamma-1) \eta}{(5-\gamma)(3-\gamma)(\gamma-2) \tilde{r}^{\gamma-2}}-\frac{\eta \tilde{R}^{2}}{(5-\gamma) \tilde{r}^{\gamma}}, \quad(\gamma \neq 2), \\
\frac{(3-2 \eta) \ln \tilde{r}}{3}-\frac{\eta \tilde{R}^{2}}{3 \tilde{r}^{2}}, \quad(\gamma=2),
\end{array}\right.
\end{aligned}
$$

where the arguments described in Appendix A impose $\eta \leq 1 / \gamma$; the limit $\eta=1 / \gamma$ recovers the case described in Eqs. (18) and (19). The presence of a central black hole of mass $M_{\mathrm{BH}}$ can be modeled in Eq. (27) by adding a term $-\mu_{\mathrm{BH}} / \tilde{r}$, where $\mu_{\mathrm{BH}} \equiv$ $M_{\mathrm{BH}} / 4 \pi \rho_{0} a^{3}$. The density in Eq. (26) becomes prominently peanut-shaped at high values of $\eta$; this is a common property of the original model studied by Schwarzschild (1979), of the flattened Plummer sphere (Lynden-Bell 1962), and of the family of "power-law" models (Evans 1994; the family includes the Binney (1991) logarithmic potential as a special case).

For $\gamma>2$ the potential vanishes at infinity and is negative divergent at the origin. For $0<\gamma \leq 2$ an upper truncation must be applied to some integrals appearing in Eq. (6); in Eq. (27) an additive constant has then been set to zero, so that the potential diverges at infinity and vanishes at the origin. Finally, for $\gamma=2$ the model is characterized by a flat rotation curve, as the seed density distribution. In fact,

$v_{\mathrm{c}}^{2}=\frac{5-\gamma-2 \eta}{(5-\gamma)(3-\gamma) \tilde{R}^{\gamma-2}}+\frac{\mu_{\mathrm{BH}}}{\tilde{R}}$.

In the entire range $0<\gamma<3$, the models do not exhibit the repulsive behavior noted at the end of Sect. 4.1.1 for power-law tori and so, from this point of view, are physically acceptable.

The fully nonlinear (i.e., up to $\eta^{2}$ terms included) Jeans Eqs. (14) and (15) for the assumed density-potential pair can be solved without difficulty. Here, for simplicity, we use the solution up to first order in $\eta$; the analytical expressions of the intrinsic and (edge-on) projected kinematical fields are given in Appendix C. In Appendix C we also show that the projection of all the quadratic (intrinsic) kinematical profiles can be obtained explicitly even for $\mu_{\mathrm{BH}} \neq 0$. Unfortunately, the streaming velocity field cannot be obtained in explicit form when $\mu_{\mathrm{BH}} \neq 0$; for this case we can only provide asymptotic expressions for small values of the projected radius. Close to the origin, independently of the value of $\gamma$, the dynamical effects of $\mu_{\mathrm{BH}}$ are always dominant. 
For $\mu_{\mathrm{BH}}=0$, the analysis presented in Appendix $\mathrm{C}$ gives (for $3 / 2<\gamma<5 / 2$ )

$$
\begin{aligned}
M_{\mathrm{p}} \sigma_{\mathrm{a}}^{2}= & \frac{\pi^{3 / 2} \Gamma(\gamma-3 / 2) \tilde{\ell}^{5-2 \gamma}}{2(5-\gamma)(3-\gamma)(5-2 \gamma) \Gamma(\gamma)} \\
& \times\left[2(5-\gamma)+\eta\left(2 \gamma^{2}-7 \gamma-2\right)\right. \\
& \left.-\frac{4 k^{2} \eta \Gamma^{2}(3 \gamma / 4-1 / 2) \Gamma(\gamma / 2) \Gamma(\gamma)}{\Gamma^{2}(3 \gamma / 4) \Gamma(\gamma / 2-1 / 2) \Gamma(\gamma-3 / 2)}\right],
\end{aligned}
$$

where $\tilde{\ell} \equiv \sqrt{\tilde{y}^{2}+\tilde{z}^{2}}$ is the (dimensionless) circular aperture radius and $\Gamma$ is the standard Euler gamma function.

For $\mu_{\mathrm{BH}} \neq 0$ and $l \rightarrow 0$ (that is, in practice, for apertures not larger than the sphere of influence of the black hole)

$$
\begin{aligned}
& M_{\mathrm{p}} \sigma_{\mathrm{a}}^{2}=\frac{\mu_{\mathrm{BH}} \pi^{3 / 2} \Gamma(\gamma / 2+1) \tilde{\ell}^{2-\gamma}}{2 \gamma(2-\gamma) \Gamma(\gamma / 2+5 / 2)} \\
& \times\left[2(3+\gamma)-\gamma(4-\gamma) \eta-\frac{k^{2} \eta \gamma\left(\gamma^{2}-1\right) \Gamma^{2}(\gamma+1 / 4)}{\Gamma^{2}(\gamma / 2+3 / 4)}\right]
\end{aligned}
$$

for $1<\gamma<2$. Thus, scale-free oblate systems with a central black hole do have finite projected fields in the range $3 / 2<\gamma<$ 2. In Eqs. (29) and (30) $\sigma_{\mathrm{a}}^{2}$ is the aperture velocity dispersion defined as $\sigma_{\mathrm{a}}^{2}(\ell) \equiv \int_{y^{2}+z^{2} \leq \ell^{2}} \Sigma \sigma_{\text {los }}^{2} \mathrm{~d} y \mathrm{~d} z / M_{\mathrm{p}}(\ell)$, where $M_{\mathrm{p}}(\ell)$ is the projected mass within $\ell$.

By combining the two formulae above we obtain an expression for the aperture velocity dispersion which is asymptotically correct for small and large apertures when $\mu_{\mathrm{BH}} \neq 0$.

\subsubsection{Effects of rotation on the aperture velocity dispersion}

In LC03, the problem of projection effects on the tilt and thickness of the Fundamental Plane (FP) of elliptical galaxies was investigated by means of a novel approach, introduced by Bertin et al. (2002). In particular, it was shown that rotation has no significant effects on the value of the measured "central" velocity dispersion (consistent with the results obtained by van Albada et al. 1995 for cuspier models), and thus has a negligible effect on the FP tilt and thickness. However, the models used suffered from possessing unrealistically flat density profiles in their central regions. Here we illustrate how such legitimate concern can be resolved with our method, for simplicity restricting our discussion to the edge-on case, where the effect of rotation is maximum.

In Fig. 2 we show the position of our models with respect to the edge-on FP. The models are first (arbitrarily) placed on the FP (the diagonal solid line) in the isotropic case (i.e., $k=1$ ); the two dotted lines parallel to the diagonal show the 1-rms dispersion of real galaxies around the FP. We refer to the FP coefficients measured by Jørgensen et al. (1996; for Coma cluster ellipticals and $H_{0}=50 \mathrm{~km} \mathrm{~s}^{-1} \mathrm{Mpc}^{-1}$ ).

The models are then "moved" horizontally by letting their "observed" velocity dispersion $\sigma_{0}$ vary with the Satoh parameter $k$ down to $k=0$, while their scale-length and mean surface brightness are kept constant. To better illustrate our point, in Fig. 2 we place different models at different values of $R_{\mathrm{e}}$.

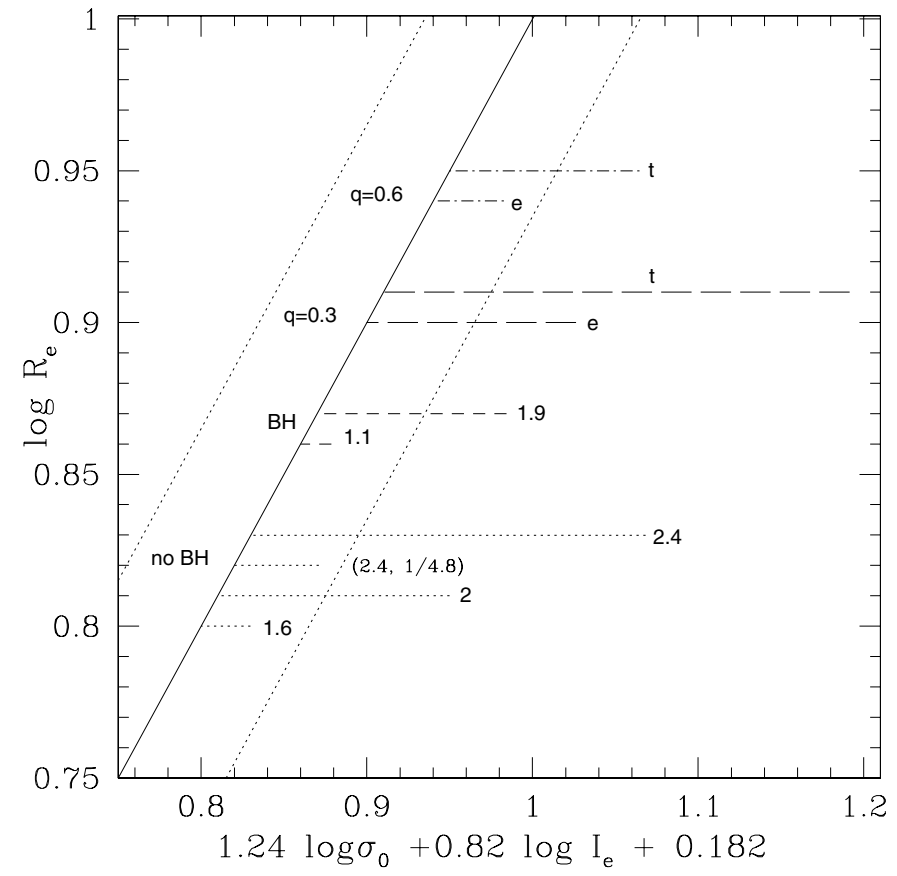

Fig. 2. Relative displacement of galaxy models induced by different amounts of rotational support with respect to the edge-on FP for different slopes $\gamma$ of the density profile and different flattenings. The two dotted lines parallel to the FP line represent the 1-rms thickness of the FP. The aperture velocity dispersion of each model is normalized to that of the corresponding isotropic rotator $(k=1)$, which is placed on the FP (solid diagonal line). Dotted and short-dashed lines (labelled by $\gamma$, lower part of the figure) represent models of Sect. 4.2 without and with a central dominant black hole, in the case of maximum flattening, except for the dotted line labeled $(2.4,1 / 4.8)$ which represents a model with smaller flattening. Long-dashed and dotted-dashed lines refer to Eq. (C11) of LC03, i.e., to the velocity dispersion of the homogeneous oblate ellipsoid $(n=0)$ calculated within an elliptical aperture with semimajor axis equal to the effective radius $R_{\mathrm{e}}$ (label "e") and over the whole model (label "t"), for two different flattenings (axial ratio $q=0.3$ and $q=0.6$ ).

Because of the scale-free nature of the models considered the relative effects are independent of the adopted aperture (cf. Eqs. (29) and (30)). In all models $\sigma_{0}$ increases for decreasing $k$, i.e., as the flattening becomes more and more supported by the azimuthal velocity dispersion. However, the displacement depends on the density slope $\gamma$ and on the model flattening. Note how cuspier models are more affected by rotation than softer ones. The effect of flattening at fixed $\gamma$ is then shown by the line labeled $(2.4,1 / 4.8)$ : for this model the density slope is $\gamma=2.4$, but the flattening is only half of the maximum allowed. The effect of rotation for models with a dominant central black hole is apparently weaker.

The behavior of cuspy models, even in the presence of a massive central black hole, is not very different from that of the Ferrers models in LC03, thus confirming the findings of van Albada et al. (1995) and the statistical results of LC03. 


\subsection{The $v / \sigma$-ellipticity plane}

One important property of a family of models characterized by different degrees of flattening, is the way it covers the socalled $v / \sigma$-ellipticity plane. In fact, such plane is a simple tool often used to investigate to what extent the observed flattening of galaxies should be ascribed to rotation (e.g., Illingworth 1977; Binney 1978). For the purpose, the observed quantities $(v / \sigma$, a measurement of the ratio of observed rotation to velocity dispersion, and the observed ellipticity) are usually compared to a curve associated with the behavior of classical spheroids (see Chandrasekhar 1969). However, such a comparison may be misleading, because (1) galaxies need not conform to the properties of classical spheroids with density stratified on homeoidal surfaces (Roberts 1962), and (2) the observations sample the central regions and may thus provide insufficient information for a proper comparison with the theoretical expectations. Indeed, Evans \& de Zeeuw (1994), in the study of their "power-law" models, noted that their nearly isotropic models are associated with points in the $v / \sigma$-ellipticity plane systematically below the curve of the classical spheroids, which is obtained from virial (and therefore integrated) quantities.

Here we address this issue by studying the behavior of the family of models introduced in Sect. 4.2. Note that, even though our models are indeed obtained from a seed density distribution stratified on homeoidal surfaces, their density distribution is not stratified on homeoids, being the sum of a spherical density distribution and a (negative) toroidal density. To stay closer to the issue of the relevant observed quantities we define $\sigma$ in terms of the aperture velocity dispersion (Eqs. (29) and (30)) and $v$ in terms of the major axis projected streaming velocity (obtained from Eq. (C8)). Note that, because of the scale-free nature of the present models, the rotational parameter is independent of the aperture radius adopted, when both the streaming velocity and the aperture velocity dispersion are measured at the same radius.

In Fig. 3 we show the model position in the ellipticityrotational parameter $(v / \sigma)$ space for edge-on, isotropic stellar systems. For the $n=0$ model described in LC03 the major axis projected rotational velocity increases linearly with radius and thus, following Evans \& de Zeeuw (1994) (footnote 2 there), we adopt as reference value of $v$ the value $u_{\mathrm{p}}$ measured at the "core" radius (i.e. at $\sqrt{3} R_{t} / 2$, where $R_{t}$ is the ellipsoid semimajor axis). The aperture velocity dispersion $\sigma$ referred to an elliptical aperture of core radius (curve "c"). It is thus clear that the rotational parameter $v / \sigma$ can stay above or below the locus of classical spheroids. In fact, it can be easily proved that for the constant density spheroid the line-of-sight velocity dispersion and the projected velocity dispersion coincide (Eq. (C.10) in LC03), and so from the projected virial theorem (e.g. Ciotti 1994) the aperture velocity dispersion over the whole object coincides with the virial velocity dispersion (in the isotropic case). Also, the streaming velocity, projected for the edge-on isotropic case, evaluated along the major axis at $\sqrt{2 / 5} R_{t}$ coincides with the rotational velocity obtained from the virial ordered kinetic energy. For example, curve " 0 " refers to the $n=0$ model where $v$ is taken at $\sqrt{2 / 5} R_{t}$, while $\sigma$ is the central projected velocity dispersion.

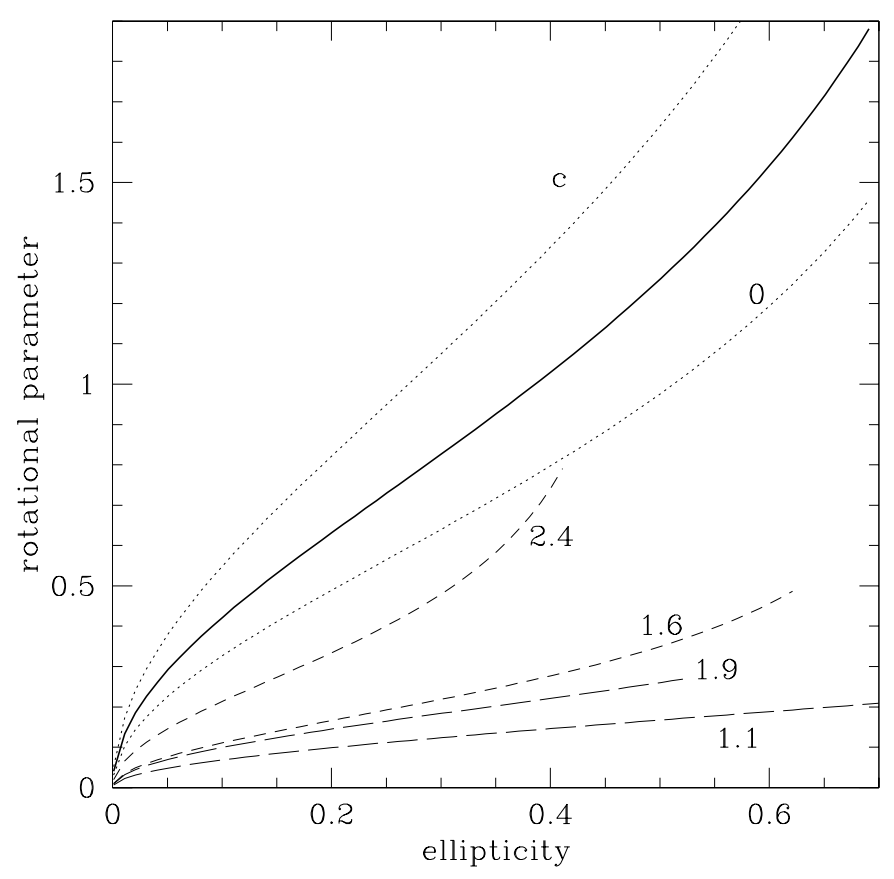

Fig. 3. The $v / \sigma$-ellipticity plane for edge-on isotropic rotators (BT87, Eq. (4.95)). Here the ellipticity is defined as the value of $\eta$. The thick solid curve is the locus of classical (oblate) spheroids. The dotted line "c" refers to the $n=0$ model in LC03, where the projected rotational velocity and the aperture velocity dispersion are taken at the "core" radius and within an elliptical aperture of semimajor axis equal to the core radius, respectively. The dotted line " 0 " refers to the same model, where the projected rotational velocity is taken at $\sqrt{2 / 5} R_{t}$ (see text), while the aperture velocity dispersion is measured at the center. Longdashed and short-dashed lines refer to our models, with and without a dominant central black hole respectively, and for different values of the density slope $\gamma$ (given by the labels) of $\rho_{0} / \mathrm{m}^{\gamma}$.

The result we wish to point out here is associated with the dashed lines, which correspond to our models with (longdashed) and without (short-dashed) a dominant central black hole, for density slopes near the limits of the acceptable range (labels near the curves give the value of $\gamma$ for the assumed $\rho=$ $\rho_{0} / m^{\gamma}$ of Sect. 4.2).

Thus, while our analysis confirms the importance of aperture effects even for the classical spheroids, we are also able to show that in general models different from classical spheroids may be found well below the classical locus, with deviations increasing at larger ellipticities.

\section{Concluding remarks}

The applications addressed in some detail in Sect. 4 are only a small sample of the many applications that can grow out of the general method introduced in this paper. Among other applications not addressed here, we may mention the following. MC04 adopt the method to calculate stellar orbits in triaxial elliptical galaxies, while Barnabé et al. (2005) investigate the properties of exploratory models for the low-rotation, extraplanar gas in spiral galaxies. Finally, we should recall that the interest in the study of toroidal structures is also motivated by the fact that centrally depressed surface brightness profiles need not 
always be associated with the projection of spherically symmetric, non-monotonic density profiles (Ciotti 2000), but may trace instead the presence of truly toroidal structures.

\section{References}

Barnabé, M., Fraternali, F., Ciotti, L., \& Sancisi, R. 2005, in Extraplanar gas, ed. R. Braun, ASP Conf. Ser., 331, 231

Bertin, G., Ciotti, L., \& Del Principe, M. 2001, A\&A, 386, 149

Binney, J. 1978, MNRAS, 183, 501

Binney, J. 1981, MNRAS, 196, 455

Binney, J., \& Tremaine, S. 1987, Galactic Dynamics (Princeton: Princeton University Press) (BT87)

Binney, J., Davies, R. L., \& Illingworth, G. D. 1990, ApJ, 361, 78

Chandrasekhar, S. 1969, Ellipsoidal figures of equilibrium (New Haven: Yale University Press)

Ciotti, L. 1994, Cel. Mech. \& Dyn. Astr., 60, 401

Ciotti, L. 2000, Astroph. Lett. Comm., 40, 85

Ciotti, L., \& Pellegrini, S. 1996, MNRAS, 279, 240

Ciotti, L., Bertin, G., \& Londrillo, P. 2004, in Plasmas in the laboratory and in the universe: new insights and new challenges, ed. G. Bertin, D. Farina, \& R. Pozzoli, AIP Conf. Proc., 703, 322

de Vaucouleurs, G. 1948, Ann. Astrophys., 11, 247

de Zeeuw, P. T., \& Carollo, C. M. 1996, MNRAS, 281, 1333

de Zeeuw, P. T., \& Merritt, D. 1983, ApJ, 267, 571

de Zeeuw, P. T., \& Pfenniger, D. 1988, MNRAS, 235, 949

Dejonghe, H. 1986, Phys. Rep., 133, 218

Dehnen, W. 1993, MNRAS, 265, 250

Evans, N. W. 1994, MNRAS, 267, 333

Evans, N. W., \& de Zeeuw, P. T. 1992, MNRAS, 257, 152

Evans, N. W., \& de Zeeuw, P. T. 1994, MNRAS, 271, 202

Ferrers, N. M. 1877, Quart. J. Pure Appl. Math., 14, 1

Fricke, W. 1952, Astron. Nachr., 280, 193

Gradshteyn, I. S., \& Ryzhik, I. M. 1980, Table of integrals, series, and products, corrected and enlarged edition (San Diego: Academic Press)

Hernquist, L., \& Quinn, P. J. 1989, ApJ, 342, 1

Hunter, C. 1975 , AJ, 80, 783

Hunter, C. 1977, AJ, 82, 271
Hunter, C., \& Qian, E. 1993, MNRAS, 262, 401

Illingworth, G. D. 1977, ApJ, 218, L43

Jackson, J. D. 1999, Classical electrodynamics, third edition (New York: Wiley)

Jaffe, W. 1983, MNRAS, 202, 995

Jeffreys, Sir H. 1970, The Earth, fifth edition (Cambridge: Cambridge University Press)

Jørgensen, I., Franx, M., \& Kjærgaard, P. 1996, MNRAS, 280, 167

Kellogg, O. D. 1953, Foundations of potential theory (New York: Dover)

Kutuzov, S. A. 1998, Astron. Lett., 24, 645

Kutuzov, S. A., \& Osipkov, L. P. 1980, Astron. Zh., 57, 28

Lanzoni, B., \& Ciotti, L. 2003, A\&A, 404, 819 (LC03)

Lee, J., \& Suto, Y. 2003, ApJ, 585, 151

Lee, J., \& Suto, Y. 2004, ApJ, 601, 599

Lynden-Bell, D. 1962, MNRAS, 123, 447

Mestel, L. 1963, MNRAS, 126, 553

Miyamoto, M., \& Nagai, R. 1975, PASJ, 27, 533

Monet, D. G., Richstone, D. O., \& Schechter, P. L. 1981, ApJ, 245, 454

Muccione, V., \& Ciotti, L. 2003, in Galaxies and Chaos, Lecture Notes on Physics, ed. G. Contopoulos, \& N. Voglis, 626, 387 (New York: Springer-Verlag) (MC03)

Muccione, V., \& Ciotti, L. 2004, A\&A, 421, 583 (MC04)

Pfenniger, D. 1984, A\&A, 134, 373

Qian, E., de Zeeuw, P. T., van der Marel, R. P., \& Hunter, C. 1995, MNRAS, 274, 602

Roberts, P. H. 1962, ApJ, 136, 1108

Satoh, C. 1980, PASJ, 32, 41

Schwarzschild, M. 1979, ApJ, 232, 236

Stäckel, P. 1890, Math. Ann., 35, 91

Tassoul, J. L. 1978, Theory of rotating stars (Princeton: Princeton University Press)

Toomre, A. 1982, ApJ, 259, 535

Tremaine, S., Richstone, D. O., Byun, Y., et al. 1994, AJ, 107, 634

van Albada, T., Bertin, G., \& Stiavelli, M. 1995, MNRAS, 276, 1255

van der Marel, R. P. 1991, MNRAS, 253, 710

van der Marel, R. P., Binney, J., \& Davies, R. L. 1990, MNRAS, 245, 582 


\section{Online Material}




\section{Ciotti and G. Bertin: Exact density-potential pairs, Online Material p 2}

\section{Appendix A: Limits on $\epsilon$ and $\eta$}

When considering homeoidal expansions we have to impose that the density distribution thus obtained be positive-definite, which then sets limits on the maximum values of $\epsilon$ and $\eta$ as a function of the seed density profile. Without loss of generality we take $\epsilon \leq \eta$. In the case of the expansion at fixed total mass (see MC04, Eq. (C.1)) we require

$1+\epsilon+\eta+\frac{\epsilon y^{2}+\eta z^{2}}{r^{2}} \frac{\mathrm{d} \ln \rho(r)}{\mathrm{d} \ln r} \geq 0$.

If we assume that $\rho(r)$ is a non-increasing function of $r$, by defining $A_{\mathrm{M}} \equiv \sup _{[0, \infty[}|\mathrm{d} \ln \rho(r) / \mathrm{d} \ln r| \geq 0$, the inequality above is then satisfied when

$\epsilon \geq\left(A_{\mathrm{M}}-1\right) \eta-1$

because the factor $\left(\epsilon y^{2}+\eta z^{2}\right) / r^{2}$ attains its maximum value $\eta$ on the $z$ axis. Clearly, if the seed density profiles are too steep little room is left for the expansion procedure. In the unconstrained expansion (Eq. (4)), a similar analysis for $\epsilon \leq \eta \leq 1$ leads to the condition $1 \geq A_{\mathrm{M}} \eta$.

To illustrate these conclusions, let us consider the density profile

$\tilde{\rho}=\frac{1}{m^{a}\left(1+m^{b}\right)^{c}}$

with $a \geq 0, b \geq 0, c \geq 0$, for which $A_{\mathrm{M}}=a+b c$. Thus, in the unconstrained expansion we have to require $\eta \leq 1 /(a+$ $b c$ ), while in the oblate case at fixed total mass the relevant condition is $\eta \leq 1 /(a+b c-1)$. Thus, for a power-law model $(c=0), 0 \leq \eta \leq 1 / a$.

A particularly interesting case of expansion at fixed total mass is represented by the so-called $\gamma$-models (Dehnen 1993; Tremaine et al. 1994), for which $a=\gamma, b=1, c=4-\gamma$. The $\gamma$-models received much attention not only for their analytical simplicity, but also because the projected density distribution of the $\gamma=2$ model (Jaffe 1983) is well fitted by the de Vaucouleurs (1948) $R^{1 / 4}$ law. Curiously, the positivity condition for the oblate case is $0 \leq \eta \leq 1 / 3$, i.e., the expansion must be limited to models rounder than E7. For the $\gamma$ models we have $\mathcal{M}=1 /(3-\gamma)$, and so, from Eq. (C.1) of MC04

$\varrho=\frac{1+\epsilon+\eta}{\tilde{r}^{\gamma}(1+\tilde{r})^{4-\gamma}}-\frac{\left(\epsilon \tilde{y}^{2}+\eta \tilde{z}^{2}\right)(4 \tilde{r}+\gamma)}{\tilde{r}^{2+\gamma}(1+\tilde{r})^{5-\gamma}}$,

with

$$
\begin{aligned}
& \tilde{\phi}_{0}=\left\{\begin{array}{l}
\frac{1}{(2-\gamma)(3-\gamma)}\left[\left(\frac{\tilde{r}}{1+\tilde{r}}\right)^{2-\gamma}-1\right], \quad(\gamma \neq 2) ; \\
\ln \left(\frac{\tilde{r}}{1+\tilde{r}}\right), \quad(\gamma=2)
\end{array}\right. \\
& \tilde{\phi}_{1}=\left\{\begin{array}{l}
\frac{1}{3(2-\gamma)(3-\gamma)}\left[\frac{\tilde{r}^{2-\gamma}(3-\gamma+\tilde{r})}{(1+\tilde{r})^{3-\gamma}}-1\right] \\
-\frac{2 F_{1}(4-\gamma, 5-\gamma ; 6-\gamma,-\tilde{r})}{\tilde{r}^{\gamma-2} 3(5-\gamma)}, \quad(\gamma \neq 2) ; \\
\frac{\tilde{r}-2}{3 \tilde{r}^{2}}+\frac{1}{3} \ln \left(\frac{\tilde{r}}{1+\tilde{r}}\right)+\frac{2 \ln (1+\tilde{r})}{3 \tilde{r}^{3}}, \quad(\gamma=2)
\end{array}\right.
\end{aligned}
$$
by solving the (dimensionless) Poisson equation $\nabla^{2} \phi_{\mathrm{S}}=\rho_{\mathrm{S}}$

$\tilde{\phi}_{2}=\left\{\begin{array}{l}\frac{{ }_{2} F_{1}(4-\gamma, 5-\gamma ; 6-\gamma,-\tilde{r})}{\tilde{r}^{\gamma}(5-\gamma)}, \quad(\gamma \neq 2) ; \\ \frac{2+\tilde{r}}{\tilde{r}^{4}(1+\tilde{r})}-\frac{2 \ln (1+\tilde{r})}{\tilde{r}^{5}}, \quad(\gamma=2) .\end{array}\right.$

The case $\gamma=1$ was adopted by MC03 and MC04, and similar formulae can also be obtained for the so-called modified Jaffe profile, $\rho \propto 1 / m^{2}\left(1+m^{2}\right)$. For $\gamma=0,1,2$ the Jeans equations can be solved explicitly, even in the presence of a central black hole, with functions no more complicated than standard hypergeometric functions.

\section{Appendix B: Relation with expansions in spherical harmonics}

Equation (7) can be recovered also from a study based on spherical harmonics. Let us assume that the density distribution is given in spherical coordinates by $\rho=-\rho_{0} r \tilde{\rho}^{\prime}(r) \cos ^{2} \vartheta$. In a standard expansion in spherical harmonics $\rho(\boldsymbol{x})=$ $\sum_{l=0}^{\infty} \sum_{m=-l}^{l} D_{l m}(r) Y_{l m}(\vartheta, \varphi)$ (e.g., Jackson 1999); for our case we thus have

$D_{l m}(r)=-\rho_{0} r \tilde{\rho}^{\prime}(r) \frac{\sqrt{4 \pi}}{3} \delta_{m 0}\left[\delta_{l 0}+\frac{2}{\sqrt{5}} \delta_{l 2}\right]$.

Some algebra then leads to prove that the associated potential is indeed the one recorded in Eq. (7).

In the seminal paper describing the numerical set-up of the "Schwarzschild method" for the construction of triaxial models of elliptical galaxies (Schwarzschild 1979), the (dimensionless) density distribution was chosen of the form

$\rho_{\mathrm{S}}=F(r)-G(r) \frac{2 z^{2}-x^{2}-y^{2}}{2 r^{2}}+3 H(r) \frac{x^{2}-y^{2}}{r^{2}}$,

where $F(r)=\left(1+r^{2}\right)^{-3 / 2}$, the two non-spherical terms have factors of the form of $l=2$ spherical harmonics, and $H$ and $G$ are functions prescribed implicitly from separate considerations and then calculated numerically. Given the above choice of $\rho_{\mathrm{S}}$, the related potential can be written as

$\phi_{\mathrm{S}}=U(r)-V(r) \frac{2 z^{2}-x^{2}-y^{2}}{2 r^{2}}+3 W(r) \frac{x^{2}-y^{2}}{r^{2}}$

and then Schwarzschild obtained $V=V[G]$ and $W=W[H]$ numerically. Here we note that $V[G]$ and $W[H]$ are also available in closed integral representation for a generic $F(r)$ (starting from Eqs. (8) and (9) with the condition $\alpha+\beta+\gamma=0$ ).

Several authors constructed triaxial density-potential pairs starting from Eqs. (B2) and (B3), through a potential-priority variation of the Schwarzschild method (e.g., de Zeeuw \& Merritt 1983; Hernquist \& Quinn 1989; de Zeeuw \& Carollo 1996). We wish to emphasize that the approach based on homeoidal expansions developed in this paper to construct analytically tractable density-potential pairs leads to new solutions. For example, models constructed from an expansion of triaxial $\gamma$-models are different from those studied by de Zeeuw \& Carollo (1996). 


\section{Appendix C: Solutions of the Jeans equations for low-flattening, scale-free axisymmetric models}

Here we record the expressions for the intrinsic and projected kinematical profiles associated with the density profile in Eq. (26), derived from the Jeans equations under the assumption that the underlying DF depends on $E$ and $J_{z}$. We adopt the same normalization procedure mentioned at the beginning of Sect. 4.

We first note that for $\gamma>1$ the edge-on projected density ("surface brightness") is

$\Sigma=\frac{\sqrt{\pi} \Gamma(\gamma / 2-1 / 2)}{\Gamma(\gamma / 2) \tilde{\ell}^{\gamma-1}}\left[1-\frac{(\gamma-1) \eta \tilde{z}^{2}}{\tilde{\ell}^{2}}\right]$

and the mass contained within the projected radius $\ell=$ $\sqrt{y^{2}+z^{2}}$ ("integrated luminosity") is

$M_{\mathrm{p}}=\frac{\pi^{3 / 2}(2+\eta-\gamma \eta) \Gamma(\gamma / 2-1 / 2)}{(3-\gamma) \Gamma(\gamma / 2)} \tilde{\ell}^{3-\gamma}$.

Integration of Eq. (14), truncated to first order in $\eta$, gives

$$
\begin{aligned}
\varrho \sigma^{2}= & \frac{1}{(5-\gamma)(3-\gamma) \tilde{r}^{2(\gamma-1)}}\left[\frac{5-\gamma-4 \eta}{2(\gamma-1)}-\frac{(4-\gamma) \eta \tilde{z}^{2}}{\tilde{r}^{2}}\right] \\
& +\frac{\mu_{\mathrm{BH}}}{(3+\gamma) \tilde{r}^{1+\gamma}}\left(\frac{3+\gamma-2 \gamma \eta}{1+\gamma}-\frac{\gamma \eta \tilde{z}^{2}}{\tilde{r}^{2}}\right) .
\end{aligned}
$$

Similarly, from Eq. (15),

$\varrho\left(\overline{v_{\varphi}^{2}}-\sigma^{2}\right)=\frac{2 \eta \tilde{R}^{2}}{\tilde{r}^{2 \gamma}}\left[\frac{1}{(5-\gamma)(3-\gamma)}+\frac{\gamma \mu_{\mathrm{BH}}}{(3+\gamma) \tilde{r}^{3-\gamma}}\right]$.

Therefore, for spherical systems $(\eta=0)$, the quantity above vanishes. Finally, to leading order in $\eta$

$$
\begin{aligned}
\varrho u_{\varphi} & =\sqrt{\varrho \times \varrho u_{\varphi}^{2}} \\
& \sim \frac{k \sqrt{2 \eta} \tilde{R}}{\tilde{r}^{3 \gamma / 2}} \sqrt{\frac{1}{(5-\gamma)(3-\gamma)}+\frac{\gamma \mu_{\mathrm{BH}}}{(3+\gamma) \tilde{r}^{3-\gamma}}} .
\end{aligned}
$$

The relevant projected kinematical quantities are obtained from the formulae described in Sect. 3. Accordingly,

$$
\begin{aligned}
\Sigma V_{\mathrm{p}}^{2}= & \frac{2 \eta k^{2} \sqrt{\pi} \tilde{y}^{2}}{\tilde{\ell}^{2 \gamma-1}} \times\left[\frac{\Gamma(\gamma-1 / 2)}{(5-\gamma)(3-\gamma) \Gamma(\gamma)}\right. \\
& \left.+\frac{\gamma \mu_{\mathrm{BH}}}{\tilde{\ell}^{3-\gamma}} \frac{\Gamma(\gamma / 2+1)}{2 \Gamma(\gamma / 2+5 / 2)}\right],
\end{aligned}
$$

and, for $\gamma>3 / 2$,

$$
\begin{aligned}
\Sigma \sigma_{\mathrm{p}}^{2}= & \frac{\sqrt{\pi} \Gamma(\gamma-1 / 2)}{(5-\gamma)(3-\gamma) \Gamma(\gamma) \tilde{\ell}^{2 \gamma-3}}\left[\frac{5-\gamma-4 \eta}{2 \gamma-3}-\frac{(4-\gamma) \eta \tilde{z}^{2}}{\tilde{\ell}^{2}}\right] \\
& +\frac{\mu_{\mathrm{BH}} \sqrt{\pi} \Gamma(\gamma / 2+1)}{2 \Gamma(\gamma / 2+5 / 2) \tilde{\ell}^{\gamma}}\left(\frac{3+\gamma-2 \gamma \eta}{\gamma}-\frac{\gamma \eta \tilde{z}^{2}}{\tilde{\ell}^{2}}\right) \\
& +\frac{1-k^{2}}{k^{2}} \Sigma V_{\mathrm{p}}^{2} .
\end{aligned}
$$

Unfortunately, when $\mu_{\mathrm{BH}} \neq 0$ no explicit expression is available for the quantities $\Sigma u_{\mathrm{p}}$ and $\Sigma u_{\mathrm{p}}^{2}$. This latter quantity is obtained by expanding to lowest order in $\eta$ the function $\left(\Sigma u_{\mathrm{p}}\right)^{2} / \Sigma$. In two interesting cases, namely when $\mu_{\mathrm{BH}}=0$ and when $\mu_{\mathrm{BH}} \neq 0$ and $\ell \rightarrow 0$, asymptotic expressions can be obtained from the projection integral by substituting $\tilde{x}^{2}=\tilde{r}^{2}-\tilde{\ell}^{2}$ and then by changing the integration variable to $\tilde{r} / \tilde{\ell}$. One then finds

$\Sigma u_{\mathrm{p}}=$

$$
-\left\{\begin{array}{l}
\frac{\sqrt{2 \pi \eta} k \tilde{y}}{\tilde{\ell}^{3 \gamma / 2-1}} \frac{\Gamma(3 \gamma / 4-1 / 2)}{\sqrt{(5-\gamma)(3-\gamma)} \Gamma(3 \gamma / 4)}, \quad\left(\mu_{\mathrm{BH}}=0\right) ; \\
\frac{\sqrt{2 \pi \eta \mu_{\mathrm{BH}}} k \tilde{y}}{\tilde{\ell}^{\gamma+1 / 2}} \frac{\sqrt{\gamma} \Gamma(\gamma / 2+1 / 4)}{\sqrt{3+\gamma} \Gamma(\gamma / 2+3 / 4)}, \\
\left(\mu_{\mathrm{BH}} \neq 0, \tilde{\ell} \rightarrow 0\right),
\end{array}\right.
$$

and

$\Sigma u_{\mathrm{p}}^{2}=$

$$
\left\{\begin{array}{r}
\frac{2 \sqrt{\pi} \eta k^{2} \tilde{y}^{2}}{\tilde{\ell}^{2 \gamma-1}} \frac{\Gamma^{2}(3 \gamma / 4-1 / 2) \Gamma(\gamma / 2)}{(5-\gamma)(3-\gamma) \Gamma^{2}(3 \gamma / 4) \Gamma(\gamma / 2-1 / 2)}, \\
\frac{2 \sqrt{\pi} \eta \mu_{\mathrm{BH}} k^{2} \tilde{y}^{2}}{\tilde{\ell}^{2+\gamma}} \frac{\gamma \Gamma^{2}(\gamma / 2+1 / 4) \Gamma(\gamma / 2)}{(3+\gamma) \Gamma^{2}(\gamma / 2+3 / 4) \Gamma(\gamma / 2-1 / 2)}, \\
\left(\mu_{\mathrm{BH}} \neq 0, \tilde{\ell} \rightarrow 0\right) .
\end{array}\right.
$$

Near the origin, the black hole contribution to the various intrinsic and projected fields dominates for any value of $\gamma$. 